Document downloaded from:

http://hdl.handle.net/10251/157367

This paper must be cited as:

Alegre Gil, MC. (2019). The weak topology in finite dimensional asymmetric normed spaces.

Topology and its Applications. 264:455-461. https://doi.org/10.1016/j.topol.2019.06.047

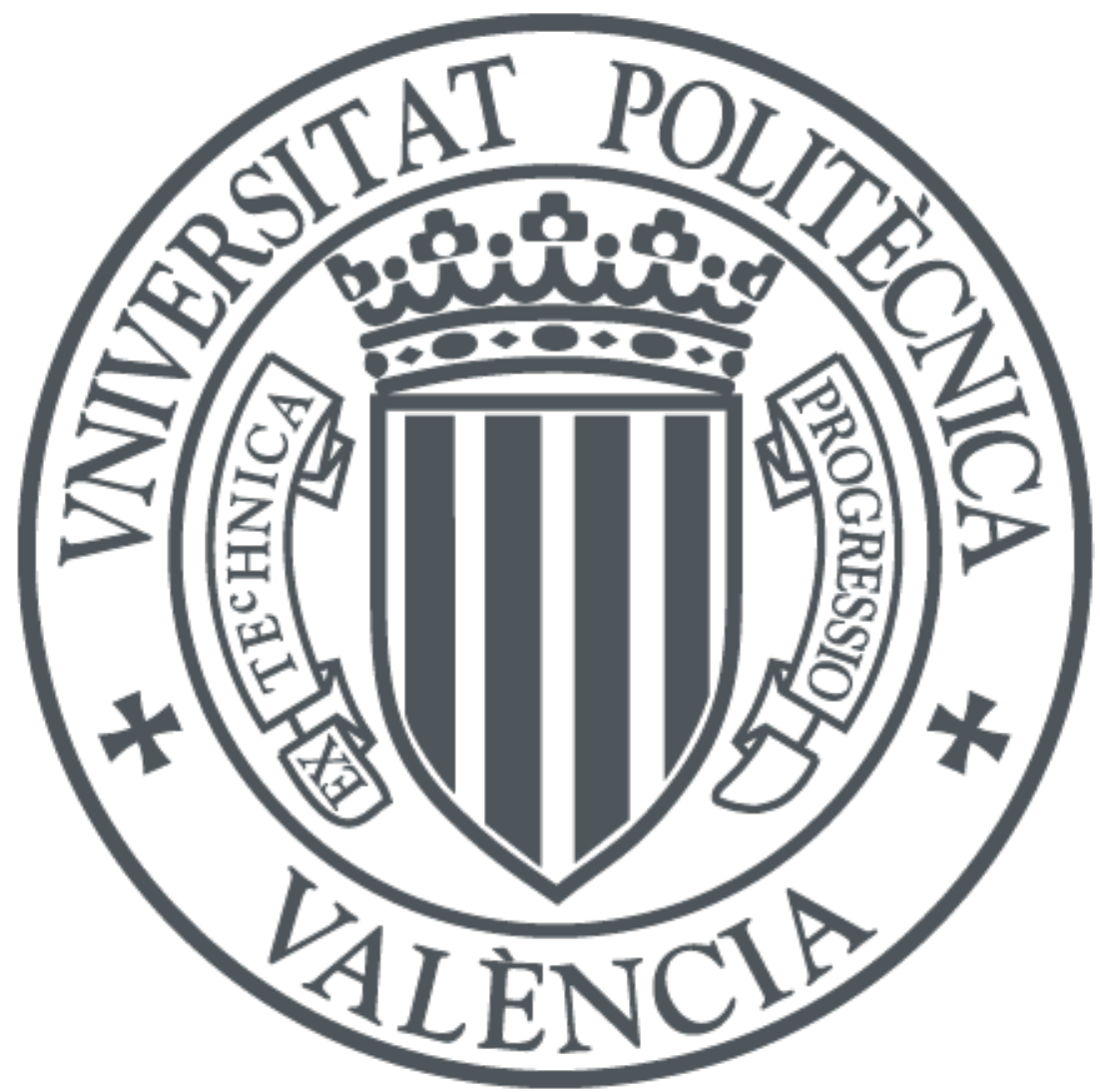

The final publication is available at

https://doi.org/10.1016/j.topol.2019.06.047

Copyright Elsevier

Additional Information 


\title{
The weak topology in finite dimensional asymmetric normed spaces *
}

\author{
Carmen Alegre
}

\begin{abstract}
In this paper we show that, in contrast to what happens in a normed space, the weak topology of a finite dimensional asymmetric normed space is not necessarily the same as the topology of the asymmetric norm. We provide a class of finite dimensional asymmetric normed spaces where both topologies coincide. We also prove that the weak topology of an infinite dimensional asymmetric normed space is strictly coarser than the topology of the asymmetric norm.
\end{abstract}

\section{Introduction}

In [6] the authors introduced different weak topologies in the context of the asymmetric normed spaces and they studied basic properties of these topologies. They also provided an interpretation of the obtained results in the context of the theory of Complexity Analysis of algorithms. It is well known that the weak topology of a normed space coincides with the topology of the norm if and only if the space is finite dimensional. In this paper we examine whether this result can be extended to the framework of asymmetric normed spaces.

We start by recalling several notions and properties of the theory of asymmetric normed spaces. Our basic reference is [2].

Let $X$ be a real linear space. A function $q: X \rightarrow \mathbb{R}^{+}$, is an asymmetric norm on $X$ if for all $x, y \in X$ and $r \in \mathbb{R}^{+}$,

${ }^{*}$ Mathematics Subject Classification (2000): 54A05, 54E35, 54C08, 46A03. Key words: asymmetric norm, asymmetric lattice norm, semicontinuous function, weak topology. 
(i) $q(x)=q(-x)=0$ if and only if $x=0$.

(ii) $q(r x)=r q(x)$.

(iii) $q(x+y) \leq q(x)+q(y)$.

The pair $(X, q)$ is called asymmetric normed linear space.

If $q$ is an asymmetric norm on $\mathrm{X}$, then the function $q^{-1}$ defined on $X$ by $q^{-1}(x)=q(-x)$ is also an asymmetric norm on $X$, called the conjugate of $q$. The function $q^{s}$ defined on $X$ by $q^{s}(x)=\max \left\{q(x), q^{-1}(x)\right\}$ is a norm on $X$.

Example 1. Denote by $u$ the function defined on $\mathbb{R}$ by $u(x)=x \vee 0$ for all $x \in \mathbb{R}$. Then $u$ is an asymmetric norm on $\mathbb{R}$ such that $u^{s}$ is the Euclidean norm on $\mathbb{R}$, i.e. $\left(\mathbb{R}, u^{s}\right)$ is the Euclidean normed space $(\mathbb{R},||$.$) .$

A quasi-metric on a nonempty set $A$ is a function $d: A \times A \rightarrow \mathbb{R}^{+}$that satisfies

(1) $d(a, b)=d(b, a)=0$ if and only if $a=b$.

(2) For every $a, b, c \in A, d(a, b) \leq d(a, c)+d(c, b)$.

Each quasi-metric $d$ on $A$ generates a $T_{0}$ topology $\tau(d)$ on $A$, which has as basic open sets the d-balls,

$$
B_{d}(a, r)=\{b \in A: d(a, b)<r\}, \quad a \in A, r>0 .
$$

An asymmetric norm $q$ on a linear space $X$ induces the quasi-metric $d_{q}$ by means of the formula

$$
d_{q}(x, y):=q(y-x), \quad x, y \in X .
$$

The $d_{q}$-ball $B_{d_{q}}(x, r)$, will be simply denoted by $B_{q}(x, r)$ and the topology $\tau\left(d_{q}\right)$ will be denoted by $\tau(q)$.

Thus, the sets

$$
B_{q}(0, \varepsilon)=\{x \in X: q(x)<\varepsilon\}, \quad \varepsilon>0,
$$

define a fundamental system of neighborhoods of zero for the topology $\tau(q)$, and for all $y \in X$, the sets $B_{q}(y, \varepsilon)=y+B_{q}(0, \varepsilon)$ define a fundamental system of neighborhoods of $y$. 
If $(X, q)$ is an asymmetric normed space, by $X^{*}$ is denoted the set

$$
X^{*}=\{f:(X, q) \rightarrow(\mathbb{R}, u): f \text { is linear and continuous }\}
$$

where $(\mathbb{R}, u)$ is the asymmetric normed space given by $u(x)=x^{+}$(see Example 1).

Note that $f \in X^{*}$ if and only if $f$ is is a linear and upper semicontinuous function from $(X, q)$ into $(\mathbb{R},|\cdot|)$. It should also be noted that if $q$ is a norm on $X$ then $X^{*}$ is the classical topological dual of the normed space $(X, q)$ because in this case a linear function $f$ is upper semicontinuous if and only if it is continuous.

The following proposition provides a useful characterization of continuity that will be used in this paper.

Proposition 1. (Corollary 1 of [1]) If $(X, q)$ is an asymmetric normed space then $f \in X^{*}$ if and only if there exists $M>0$ such that $f(x) \leq M q(x)$ for all $x \in X$.

If $(X, q)$ is an asymmetric normed space, by $X^{s *}$ is denoted the topological dual space of the normed space $\left(X, q^{s}\right)$, i.e.,

$$
X^{s *}=\left\{f:\left(X, q^{s}\right) \rightarrow(\mathbb{R},|\cdot|): f \text { is linear and continuous }\right\}
$$

The set $X^{*}$ is not necessarily a linear space, but it is a cone of $X^{s *}$. The set $X^{*}$ is called the dual space of $(X, q)$. More information about these spaces can be found in [1], [2] and [7].

An important class of asymmetric normed spaces are those where the asymmetric norm is given by a lattice norm. If $(X,\|\cdot\|, \leq)$ is a normed lattice, the function $q$ defined on $X$ by $q(x)=\left\|x^{+}\right\|$, with $x^{+}=x \vee 0$, is an asymmetric norm on $X$ and the norm $q^{s}$ is equivalent to the norm $\|\cdot\|$ (see, for example, $[1,5])$. We shall refer to $q$ as an asymmetric lattice norm on $X$. It is noted that in this class of spaces $X^{*}$ is the positive cone of $X^{s *}$.

It is well known that all norms in a finite dimensional space are equivalent, and so all the asymmetric lattice norms defined by this procedure are equivalent too. In addition, if $(X,\|\cdot\|, \leq)$ is a finite dimensional normed lattice then $X$ and $\mathbb{R}^{n}$ are order isomorphic if we consider in $\mathbb{R}^{n}$ the coordinatewise order (see Corollary 1 of Theorem 3.9 and Proposition 5.2 of [12]). Thus, there exists a linear bijection $T: \mathbb{R}^{n} \rightarrow X$ such that $x \leq y$ if and only if $T(x) \leq T(y)$. If we define in $\mathbb{R}^{n}$ the norm $\|x\|=\|T x\|$ then $T$ is a lattice isometry, therefore, $X$ and $\mathbb{R}^{n}$ are identical from the point of view of Riesz normed spaces. 


\section{The weak topology of $(X, q)$.}

Let $(X, q)$ an asymmetric normed space. The weak topology induced by $q$ (weak positive topology in [6]) for $X$, is the one that has as a base of neighborhoods of the origin the following sets

$$
V_{f_{1}, f_{2}, \ldots, f_{n}}(0, \epsilon)=\left\{x \in X: f_{1}(x)<\epsilon, f_{2}(x)<\epsilon, \ldots, f_{n}(x)<\epsilon\right\}
$$

with $f_{1}, f_{2}, \ldots, f_{n} \in X^{*}, \epsilon>0, n \in \mathbb{N}$.

A basis of neighborhoods for an element $y \in X$ is obtained by:

$$
V_{f_{1}, f_{2}, \ldots, f_{n}}(y, \epsilon)=y+V_{f_{1}, f_{2}, \ldots, f_{n}}(0, \epsilon)
$$

The weak topology induced by $q$ will be denoted by $\tau_{\text {weak }}(q)$ ( it is denoted by $\tau_{\text {weak }}^{+}$in $[6]$ ).

By definition, it is obvious that $\tau_{\text {weak }}(q) \subseteq \tau(q)$. Moreover, $\tau_{\text {weak }}(q)$ is the coarser topology on $X$ under which all the elements of $X^{*}$ are upper semicontinuous functions. Note that if $q$ is a norm on $X$, then $\tau_{\text {weak }}(q)$ is the classical weak topology on $X$.

In finite dimensional normed spaces the weak topology and the norm topology coincide. The following example shows that this result can not be extended to the asymmetric case.

Example 2. In $\mathbb{R}^{2}$ we consider the asymmetric norm

$$
q((x, y))=\frac{-y+\sqrt{4 x^{2}+y^{2}}}{2} .
$$

If $f \in\left(\mathbb{R}^{2}\right)^{*}$ then there exists $(a, b) \in \mathbb{R}^{2}$ such that $f((x, y))=a x+b y$ and there exists $M>0$ such that $f((x, y)) \leq M q((x, y))$ for all $(x, y) \in \mathbb{R}^{2}$. Since $q((0,1))=0$ we have that $b=f((0,1)) \leq 0$. On the other hand, if $g((x, y))=x$ then $g$ and $-g$ are not in $\left(\mathbb{R}^{2}\right)^{*}$. Indeed, if $g \in\left(\mathbb{R}^{2}\right)^{*}$ then there exists $\delta>0$ such that $\left.g\left(B_{q}(0, \delta)\right) \subseteq\right] \infty, 1\left[\right.$. Nevertheless, $\left(2, \frac{16-\delta^{2}}{2 \delta}\right) \in B_{q}(0, \delta)$ because $q\left(\left(2, \frac{16-\delta^{2}}{2 \delta}\right)\right)=\delta / 2$ and $g\left(\left(2, \frac{16-4 \delta^{2}}{2 \delta}\right)\right)=2$. Similarly, it is proved that $-g \notin\left(\mathbb{R}^{2}\right)^{*}$. Therefore,

$$
\left(\mathbb{R}^{2}\right)^{*} \subseteq\left\{(a, b) \in \mathbb{R}^{2}: b<0\right\} \cup\{(0,0)\} .
$$

Now we will prove that $\tau_{\text {weak }}(q)$ is strictly coarser than $\tau(q)$. Suppose that $\tau_{\text {weak }}(q)=\tau(q)$. Let $B=\{(x, y): q((x, y)) \leq 1\}$. It is easy to prove that

$$
B=\left\{(x, y): y \geq x^{2}-1\right\} \text {. }
$$


Since $B$ is a neighborhood of zero for the topology $\tau(q)$ there exist $\left(a_{1}, b_{1}\right)$, $\left(a_{2}, b_{2}\right), \ldots,\left(a_{n}, b_{n}\right) \in\left(\mathbb{R}^{2}\right)^{*} \backslash\{(0,0)\}$ and $\varepsilon>0$ such that $\bigcap_{i=1}^{n} V_{i} \subseteq B$, where

$$
\begin{gathered}
V_{i}=\left\{(x, y): a_{i} x+b_{i} y \leq \epsilon\right\}=\left\{(x, y): y \geq \frac{-a_{i}}{b_{i}} x+\frac{\varepsilon}{b_{i}}\right\} . \\
\text { If } m=\max \left\{\left|\frac{a_{i}}{b_{i}}\right|: i=1, \ldots, n\right\}, \text { then } \\
S=\{(x, y): x \geq 0, y \geq m x\} \subseteq \bigcap_{i=1}^{n} V_{i} \subseteq B .
\end{gathered}
$$

If $m=0$ there is clearly a contradiction because $S$ is not included in $B$. If $m>0$, then

$$
\left(m+\sqrt{m^{2}+4}, m^{2}+m \sqrt{m^{2}+4}\right) \in S,
$$

nevertheless $\left(m+\sqrt{m^{2}+4}, m^{2}+m \sqrt{m^{2}+4}\right) \notin B$, because

$$
\left(m+\sqrt{m^{2}+4}\right)^{2}-1>m^{2}+m \sqrt{m^{2}+4} .
$$

The next result provides a class of finite dimensional asymmetric normed spaces where the norm topology and the weak topology coincide.

Theorem 1. If $(X,\|\cdot\|, \leq)$ is a finite normed lattice and $q(x)=\left\|x^{+}\right\|$then $\tau(q)=\tau_{\text {weak }}(q)$.

Proof. We can suppose without loss of generality that $X=\mathbb{R}^{n}$. We must show that $B_{q}(0, \epsilon)$ is a neighborhood of the origin in $\tau_{\text {weak }}(q)$ for all $\epsilon>0$. Let $\left\{e_{i}\right\}_{i=1}^{n}$ the canonical basis of $\mathbb{R}^{n}$ and let $\left\{e_{i}^{*}\right\}_{i=1}^{n}$ its dual basis, i.e., $e_{i}^{*}\left(e_{j}\right)=\delta_{i, j}$. Since $e_{i}^{*}$ is continuous in $\left(\mathbb{R}^{n},\|\cdot\|\right)$ and $e_{i}^{*} \geq 0$, by Corollary 3 of [1], we have that $e_{i}^{*} \in\left(\mathbb{R}^{n}\right)^{*}$ for $i=1 \ldots, n$.

Let $M=\max \left\{\left\|e_{1}\right\|, \ldots,\left\|e_{n}\right\|\right\}$ and let $x \in V_{e_{1}^{*}, e_{2}^{*}, \ldots, e_{n}^{*}}(0, \epsilon / n M)$. If $x=$ $\left(x_{1}, x_{2}, \ldots, x_{n}\right)$, we have that $e_{i}^{*}(x)=x_{i}<\epsilon / n M$ for $i=1,2, \ldots, n$. Then

$$
q(x)=\left\|\left(x_{1}^{+}, x_{2}^{+}, \ldots, x_{n}^{+}\right)\right\| \leq \sum_{i=1}^{n} x_{i}^{+}\left\|e_{i}\right\|<\sum_{i=1}^{n} \epsilon M / n M=\epsilon .
$$

Therefore, $V_{e_{1}^{*}, e_{2}^{*}, \ldots, e_{n}^{*}}(0, \epsilon / n M) \subseteq B_{q}(0, \epsilon)$. 
In the light of this result it is natural to wonder whether the equality of the two topologies is only fulfilled if the asymmetric norm is an asymmetric lattice norm. The example below shows that this is not the case.

Example 3. In $\mathbb{R}^{2}$ we consider the asymmetric norm

$$
q((x, y))=\max \left\{|x|, y^{+}\right\} .
$$

This asymmetric norm is not an asymmetric lattice norm. Indeed, if it were so the set $\{(x, y): x \leq 0, y \leq 0\}$ is included in $B_{q}(0,1)$ and this is not true. Is easy to prove that $\tau(q)$ is strictly finer than the topology in $\mathbb{R}^{2}$ defined by an asymmetric lattice norm.

Now we prove that $f$ is an element of the dual space of $\left(\mathbb{R}^{2}, q\right)$ if and only if $f((x, y))=a x+b y$ with $b \geq 0$.

If $b \geq 0$ then

$$
f((x, y))=a x+b y \leq|a||x|+b y^{+} \leq(|a|+b) q((x, y)),
$$

therefore $f \in\left(\mathbb{R}^{2}\right)^{*}$. Conversely, if $f \in\left(\mathbb{R}^{2}\right)^{*}$, there exists $M>0$ such that $f((x, y))=a x+b y \leq M q((x, y))$ for all $(x, y) \in \mathbb{R}^{2}$. Then $-b=f((0,-1)) \leq$ $M q((0,-1))=0$, hence, $b \geq 0$.

Thus, the functions $f_{1}((x, y))=x, f_{2}((x, y))=-x$ and $f_{3}((x, y))=y$ are in $X^{*}$ and

$$
V_{f_{1}, f_{2}, f_{3}}(0, \epsilon) \subseteq B_{q}(0, \epsilon)
$$

for all $\epsilon>0$. Therefore $\tau(q)=\tau_{\text {weak }}(q)$.

Next, we prove that, as in normed spaces, the weak topology of an infinite dimensional asymmetric normed space is strictly coarser than the topology of the asymmetric norm. The proof is analogous to that for normed spaces.

Lemma 1. (Lemma 5, Chap.II of [11]) Let $X$ be a real vector space. If $f, g_{1}, g_{2}, \ldots, g_{n}$ are linear functions of $X$, then $f$ is a linear combination of $g_{1}, g_{2}, \ldots, g_{n}$ or there is an element a of $X$ with $f(a)=1$ and $g_{1}(a)=g_{2}(a)=$ $\ldots=g_{n}(a)=0$.

Proposition 2. If $(X, q)$ is an infinite dimensional asymmetric normed space then $\tau_{\text {weak }}(q)$ is strictly coarser than $\tau(q)$.

Proof. Suppose that $\tau_{\text {weak }}(q)=\tau(q)$. There exist $f_{1}, f_{2}, \ldots, f_{n} \in X^{*}$ and $\epsilon>0$ such that

$$
V_{f_{1}, f_{2}, \ldots, f_{n}}(0, \epsilon) \subseteq B_{q}(0,1)
$$


Then

$$
Y=\bigcap_{i=1}^{n} f_{i}^{-1}(0) \subset B_{q}(0,1) .
$$

Let us now see that $Y \neq\{0\}$. Suppose that $\bigcap_{i=1}^{n} f_{i}^{-1}(0)=\{0\}$. If $f$ a linear function of $X$, by previous lemma, $f$ is a linear combination of $f_{1}, f_{2}, \ldots, f_{n}$. This implies that the algebraic dual of $X$ is finite dimensional and this is not possible because $X$ is infinite dimensional

Let $x_{0} \in Y, x_{0} \neq 0$. Then $q\left(x_{0}\right) \neq 0$ or $q\left(-x_{0}\right) \neq 0$. If $q\left(x_{0}\right) \neq 0$ then $2 x_{0} / q\left(x_{0}\right) \in Y$, therefore $2 x_{0} / q\left(x_{0}\right) \in B_{q}(0,1)$, and this is a contradiction because $q\left(2 x_{0} / q\left(x_{0}\right)\right)=2$.

If $q\left(-x_{0}\right) \neq 0$, the proof is similar.

Next, we study the weak convergence in asymmetric normed spaces. Let us recall that a sequence $\left\{x_{n}\right\}$ in the asymmetric normed space $(X, q)$ is $q$-convergent to $x \in X$ if $\left\{q\left(x_{n}-x\right)\right\} \rightarrow 0$, i.e., $\left\{x_{n}\right\}$ converges to $x$ with respect to the topology $\tau(q)$.

Definition 1. Let $(X, q)$ be an asymmetric normed space and let $\left\{x_{n}\right\}$ be a sequence in $X$. We say that $\left\{x_{n}\right\}$ is weakly q-convergent to $x \in X$ if the sequence $\left\{f\left(x_{n}\right)\right\}$ is u-convergent to $f(x)$ for all $f \in X^{*}$, i.e., $\left\{\left(f\left(x_{n}\right)-\right.\right.$ $\left.f(x))^{+}\right\} \rightarrow 0$ for all $f \in X^{*}$.

Proposition 3. Let $(X, q)$ be an asymmetric normed space and let $\left\{x_{n}\right\}$ be a sequence in $X$. If $\left\{x_{n}\right\}$ is q-convergent to $x \in X$ then $\left\{x_{n}\right\}$ is q-weakly convergent to $x$.

Proof. If $f \in X^{*}$ then there exists $M>0$ such that $f(x) \leq M q(x)$, for all $x \in X$. Then

$$
\left(f\left(x_{n}\right)-f(x)\right)^{+}=\left(f\left(x_{n}-x\right)\right)^{+} \leq M q\left(x_{n}-x\right) .
$$

Since $\left\{q\left(x_{n}-x\right)\right\} \rightarrow 0$, we have that $\left\{\left(f\left(x_{n}\right)-f(x)\right)^{+}\right\} \rightarrow 0$ for all $f \in X^{*}$.

The converse of this result is not true. In infinite dimensional normed spaces there are easy examples of weakly convergent sequences which fail to be convergent. For instance, if $\left\{e_{n}\right\}$ is the standard basis of $l_{p}$ where $1<p<+\infty$ then $\left\{e_{n}\right\}$ is weakly convergent to zero and it is not convergent 
to zero with respect to the topology of the norm. Note that if $p=1$ weak convergence and norm convergence of sequences are the same (Schur's $l_{1}$ Theorem). Both notions also coincide in finite dimensional normed spaces but this does not occur in the asymmetric case. The following example illustrates this fact.

Example 4. Let $\left(\mathbb{R}^{2}, q\right)$ the asymmetric normed space given in Example 2 and let $\left\{\left(n, n^{2}\right)\right\}$ a sequence in $\mathbb{R}^{2}$. If $f \in\left(\mathbb{R}^{2}\right)^{*}$ then there exists $(a, b) \in \mathbb{R}^{2}$, with $b<0$ such that $f\left(\left(n, n^{2}\right)\right)=a n+b n^{2}$. Since $b<0$, we have that $\left\{a n+b n^{2}\right\} \rightarrow-\infty$, and so $\left\{\left(a n+b n^{2}\right)^{+}\right\} \rightarrow 0$. Therefore, the sequence $\left\{\left(n, n^{2}\right)\right\}$ is weakly q-convergent to $(0,0)$. This sequence is not q-convergent to $(0,0)$ because

$$
q\left(\left(n, n^{2}\right)\right)=\frac{-n+\sqrt{4 n^{2}+n^{4}}}{2} \geq \frac{-n+\sqrt{4 n^{2}+n^{2}}}{2}=\frac{(\sqrt{5}-1) n}{2},
$$

and this implies that $\left\{q\left(n, n^{2}\right)\right\} \rightarrow \infty$.

The next proposition characterizes weak $q$-convergence in terms of $\tau_{\text {weak }}(q)$. We omit the proof because it follows closely the classical one.

Proposition 4. Let $(X, q)$ be an asymmetric normed space and let $\left\{x_{n}\right\}$ be a sequence in $X$. Then, $\left\{x_{n}\right\}$ is q-weakly convergent if and only if it is convergent with respect the topology $\tau_{\text {weak }}(q)$.

Final remark. Recently, several properties of finite dimensional asymmetric normed spaces spaces have been investigated in a series of papers $([4,8,9,10])$. The fact that the asymmetric norm is right bounded plays a crucial role in the study of many of these properties. An asymmetric norm $q$ is said to be right bounded if there exists $r>0$ such that $r B_{q} \subseteq B_{q^{s}}+\theta_{0}$, where $B_{q}=\{x \in X: q(x) \leq 1\}$ and $\theta_{0}=\{x \in X: q(x)=0\}$. The asymmetric lattice norms are right bounded and so also is the asymmetric norm of Example 3, nevertheless the asymmetric norm of Example 2 is not ([3]). These results suggest the following question.

Question. Let $(X, q)$ be a finite dimensional asymmetric normed space. $\tau(q)=\tau_{\text {weak }}(q)$ if and only if $q$ is right bounded? 


\section{References}

[1] C. Alegre, Continuous operator on asymmetric normed spaces, Acta Math. Hungar. 122 (2009), 357-372.

[2] S. Cobzas, Functional Analysis in Asymmetric Normed Spaces, Birkhauser, Basel, 2013.

[3] J. Conradie Asymmetric norms , cones and partial orders Topology Appl. 193 (2015), 100-115.

[4] J. Conradie and M.D. Mabula, Completeness, precompacteness and compactness in finite-dimensional asymmetrically normed lattices, Topology Appl. 160 (2013), 2012-2024.

[5] J. Ferrer, V. Gregori and C. Alegre Quasi-uniform structures in linear lattices. Rocky Mountain J. Math. 23 (1993), 877-884.

[6] L.M. García Raffi, S. Romaguera and E.A. Sánchez Pérez, Weak topologies on asymmetric normed linear spaces and non-asymptotic criteria in the theory of Complexity Analysis of algorithms, J. Anal. Appl., 2(3) (2004), 125-138.

[7] L.M. García Raffi, S. Romaguera and E.A. Sánchez-Pérez, The dual space of an asymmetric normed linear space, Quaestiones Math. 26 (2003), 83-96.

[8] N. Jonard-Pérez, E. A. Sánchez-Pérez, Compact convex sets in 2dimensional asym- metric normed spaces, Quaestiones. Math. 39(1) (2016), 73-82.

[9] N. Jonard-Pérez, E. A. Sánchez-Pérez, Extreme points and geometric aspects of compact convex sets in asymmetric normed spaces, Topology Appl. 203 (2016), 12-21.

[10] N. Jonard-Pérez, E. A. Sánchez-Pérez, Local compactness in right bounded asymmetric normed spaces, Quaestiones Math. (2018), 1-15

[11] A. Robertson and W. Robertson, Topological Vector Spaces, Cambridge University Press, (1980).

[12] H.H. Schaefer, Banach Lattices and Positive Operators, Springer, 1974. 
Instituto de Matemática Pura y Aplicada.

Universidad Politécnica de Valencia, 46071 Valencia, Spain.

E-mail: calegre@mat.upv.es 